\title{
Elucidating a Novel Mechanism of Extracellular Electron Uptake in Shewanella oneidensis MR-1
}

\author{
JOSHUA D. SACKETT ${ }^{1}$, LEAH R. TRUTSCHEL ${ }^{1}$, AND \\ ANNETTE R. ROWE ${ }^{1, *}$ \\ ${ }^{1}$ Department of Biological Sciences, University of Cincinnati, \\ Cincinnati, Ohio, USA 45221 \\ *Correspondence: annette.rowe@uc.edu
}

Shewanella oneidensis MR-1 is a model organism used in electrochemical and bioremediation studies due to its diverse respiratory capabilities, including dissimilatory metal reduction. Although typically studied for its capacity to deposit electrons from respiration onto solid-phase minerals and electrodes under anaerobic conditions, MR-1 is also capable of obtaining electrons from electrodes when a suitable electron acceptor is present. For example, Rowe et al. [1] demonstrated that MR-1 is capable of oxidizing a solidphase, poised cathode as a source of electrons for aerobic respiration; however, evidence suggests that extracellular electron uptake (EEU) is not exclusively a reversal of the canonical Mtr respiration pathway. To elucidate additional genes involved in EEU, we screened a whole-genome knockout library [2,3] for deficiencies in EEU. Using a threeelectrode electrochemical bioreactor system, we monitored the establishment of electrical current over time (chronoamperometry - working electrode poised at $-600 \mathrm{mV}$ vs. $\mathrm{Ag} / \mathrm{AgCl}$ ) and across a range of voltages (cyclic voltammetry - $-600 \mathrm{mV}$ to $200 \mathrm{mV}$ vs. $\mathrm{Ag} / \mathrm{AgCl}$ ). This screen resolved five mutants with diminished capacity for EEU compared to wild type, but which do not present a phenotype for anaerobic electrode reduction or aerobic respiration. Complementation of knockout mutants restored the capacity for electron uptake to that of the wild type. This presentation will focus on our work determining the physiological role of these genes in EEU. Specifically, we are employing fluorescence microscopy to evaluate the cellular localization of these hypothetical proteins under conditions of EEU. Additionally, this work will establish the effects of these gene deletions on both membrane potential and NADH$\mathrm{NAD}^{+}$redox state in live cells. We will also speak to the potential importance of this novel EEU pathway on Shewanella ecology.

[1] Rowe AR, Rajeev P, Jain A, et al. 2018. mBio 9:e0220317. https://doi .org/10.1128/mBio.02203-17.

[2] Baym M, Shaket L, Anzai I et al. 2016. Nat Commun 7, 13270. https://doi.org/10.1038/ncomms13270.

[3] Rowe AR, Salimijazi F, Trutschel L, et al. In Prep. 